\section{Can sexual dimorphism in rheumatoid arthritis be attributed to the different abundance of Gardnerella?}

Rheumatoid arthritis (RA) is a common chronic inflammatory joint disease that can cause recurrent attacks of joint pain and swelling. Progression of RA eventually causes joint destruction, ankylosis and deformity, which are associated with significant increases in socioeconomic costs and burdens. ${ }^{12}$ Epidemiological studies conducted worldwide have shown that women are more likely than men of the same age to develop RA, and that the prevalence increases with age. More importantly, relevant literature indicates that the prevalence of RA continues to increase among women. ${ }^{3}{ }^{4}$ In addition, female patients experience more severe joint dysfunction compared with male patients. ${ }^{4}$ Emerging data clearly demonstrate that women with RA have a significantly worse functional status as measured by instruments such as the 28-joint Disease Activity Score (DAS28), Health Assessment Questionnaire and visual analogue scale. ${ }^{5}$ Consequently, an understanding of the causes of this sexual dimorphism may provide new insights and guidance for the development of interventions intended to reduce the incidence of RA.

Previously, this sexual dimorphism of RA has been explained in terms of genes located on the $\mathrm{X}$ and $\mathrm{Y}$ chromosomes, sex hormones and specific physiological and psychosocial conditions. ${ }^{4}$ However, the above perspectives were accompanied by corresponding limitations. Notably, most of the relevant literature tended to elaborate from the perspective of sex hormones. Those studies focused on the effects of sex hormones on the immune system and enabled a detailed understanding of the target immune cells and immune-related genes regulated by sex hormones. Similarly, the causes of this sexual dimorphism remain equivocal.

Recent work by Kishikawa et al is expected to elucidate this phenomenon of RA. The researchers performed a genomewide association study (GWAS) to analyse the role of the gut microbiome in Japanese patients with RA. ${ }^{6}$ In this study, faecal samples were subjected to whole-genome shotgun sequencing. Through case-control phylogenetic association tests conducted using a generalised linear regression model, nine strains present at significantly higher levels of abundance in patients with RA were finally identified, namely Prevotella denticola, Gardnerella, Gardnerella vaginalis, Porphyromonas somerae, Prevotella marshii, Prevotella disiens, Bacteroides sartorii, Prevotella corporis and Prevotella amnii. ${ }^{6}$ Moreover, the authors clearly demonstrated correlations of multiple Prevotella spp beyond P. copri with the aetiology of RA. Furthermore, a gene related to oxidative stress (R6FCZ7) was more abundantly expressed in patients with RA than in healthy subjects. Moreover, by comparing the results of a RA microbial GWAS with those of a RA GWAS, the authors determined a population-specific biological pathway linking the metagenome and host genome. These results newly revealed a relationship between the gut microbiome, host genome and RA pathology, and thus represent a new reference that better clarifies the aetiology of RA. ${ }^{6}$ Certainly, this study is expected to explain the sexual dimorphism of RA because of the nine strains mentioned by Kishikawa et al, Gardnerella, or G. vaginalis, is a clinical indicator of vaginitis in female patients. This gram-negative bacillus is isolated from female vaginal secretions and is the most frequent causative organism of vaginitis. ${ }^{78}$ Consequently, might we also interpret the differential treatment of RA according to sex from the perspective of the microbiome?

We propose the following scientific hypothesis or conjecture: Gardnerella or G. vaginalis may be associated with sexual dimorphism in RA. To our knowledge, however, no previous study has clarified this phenomenon of sexual dimorphism directly from the perspective of the microbiome. Therefore, we considered whether the mechanism of bacterial vaginitis, which is closely related to Gardnerella, might provide some clues. Fortunately, a new review of Gardnerella and vaginal health revealed that Gardnerella spp are related to female vaginal infection and also appear in other types of infection. ${ }^{8}$ More notably, these other types of infections include conditions such as acute hip arthritis, hip swelling, disc space infection, discitis, spinal osteomyelitis, joint infection and reactive arthritis. ${ }^{8}$ Although these phenomena were described only in certain clinical cases, the data were sufficient to suggest an association of Gardnerella with severe RA symptoms in female patients. However, we did not know whether the above relationship was direct or indirect. The relevant literature mentioned a mutually beneficial relationship between Gardnerella and Prevotella bivia. ${ }^{9}$ More precisely, specific amino acids produced by Gardnerella could be used by P. bivia, thus enhancing the growth of the latter species. ${ }^{9}$ This led to the bold speculation of an unknown homoplastic link between certain strains of Gardnerella and Prevotella. In other words, an interaction of Gardnerella with Prevotella may contribute to the onset of RA. The different concentrations of Gardnerella in male and female humans would eventually lead to sexual dimorphism in RA. In this regard, a single strain of Gardnerella could be isolated, cultured and transplanted into germ-free mice to verify the existence of a direct relationship with RA. Prevotella should also be isolated, cultured and transplanted into germ-free mice together with Gardnerella. A single homologous Prevotella strain should also be transplanted into germ-free mice as an experimental control. Such an experiment may roughly prove that Gardnerella directly influences the onset of RA by interacting with Prevotella spp.

In addition, relevant reports have mentioned that sex hormone levels may support the expansion of some selected microbial lineages via a positive feedback mechanism, which would contribute to the sexual dimorphism phenomenon observed in autoimmune diseases. ${ }^{10}{ }^{11}$ In other words, changes in the compositions of some microbial lineages may be affected by sex hormone levels, and in turn, regulation of the composition of some microbial lineages may contribute to changes in sex hormone levels. Again, this expands our speculation that Gardnerella may also indirectly lead to sexual dimorphism in RA by influencing the levels of sex hormones.

Regardless, more specific scientific research regarding this issue is needed. Although some of the aforementioned conjectures or speculations may fail to provide further experimental verification, we still hope to discuss our ideas through this short article and hope that our colleagues in the field of RA will pay slightly more attention to Gardnerella.

\footnotetext{
Dongsong Liu, ${ }^{1,2}$ Feng Zhang, ${ }^{3,4}$ Hong Cao, ${ }^{3}$ Xuesong Wang $\oplus^{1,2}$

${ }^{1}$ Department of Orthopedics, Affiliated Hospital of Jiangnan University, Wuxi, Jiangsu, China

${ }^{2}$ Medical Colleage of Nantong University, Nantong, Jiangsu, China

${ }^{3}$ Clinical Study Center of Functional Foods, Affiliated Hospital of Jiangnan University, Wuxi, Jiangsu, China

${ }^{4}$ Wuxi Institute of Integrated Traditional Chinese and Western Medicine, Affiliated Hospital of Jiangnan University, Wuxi, Jiangsu, China
} 
Correspondence to Professor Xuesong Wang, Department of Orthopedics, Affiliated Hospital of Jiangnan University, Wuxi, Jiangsu, China; biovictorwxs@163.com

Correction notice This article has been corrected since it published Onine First. The affiliations for Feng Zhang have been updated.

Contributors None declared.

Funding This work was supported in part by the National Natural Science Foundation of China, No. 81870544 (to FZ); the Public Health Center at Jiangnan University of China, No. JUPH201808 (to XW); the Wuxi Medical and Public Health Technology Innovation and Application Project, Jiangsu Province, China, N20192025 (to XW).

Competing interests None declared.

Patient and public involvement Patients and/or the public were not involved in the design, or conduct, or reporting, or dissemination plans of this research.

Patient consent for publication Not required.

Provenance and peer review Not commissioned; internally peer reviewed. (c) Author(s) (or their employer(s)) 2020. No commercial re-use. See rights and permissions. Published by BMJ.

\section{(D) Check for updates}

To cite Liu D, Zhang F, Cao H, et al. Ann Rheum Dis Epub ahead of print: [please include Day Month Year]. doi:10.1136/annrheumdis-2020-217214

Received 25 February 2020

Accepted 29 February 2020

Ann Rheum Dis 2020;0:1-2. doi:10.1136/annrheumdis-2020-217214
ORCID iD

Xuesong Wang http://orcid.org/0000-0002-3686-333X

\section{REFERENCES}

1 Smolen JS, Aletaha D, Barton A, et al. Rheumatoid arthritis. Nat Rev Dis Primers 2018;4:18001.

2 McInnes IB, Schett G. The pathogenesis of rheumatoid arthritis. N Engl J Med 2011;365:2205-19.

3 Safiri S, Kolahi AA, Hoy D, et al. Global, regional and national burden of rheumatoid arthritis 1990-2017: a systematic analysis of the Global Burden of Disease study 2017. Ann Rheum Dis 2019:78:1463-71.

4 Kovacs WJ, Olsen NJ. Sexual dimorphism of RA manifestations: genes, hormones and behavior. Nat Rev Rheumatol 2011;7:307-10.

5 Ahlmén M, Svensson B, Albertsson K, et al. Influence of gender on assessments of disease activity and function in early rheumatoid arthritis in relation to radiographic joint damage. Ann Rheum Dis 2010;69:230-3.

6 Kishikawa T, Maeda Y, Nii T, et al. Metagenome-wide association study of gut microbiome revealed novel aetiology of rheumatoid arthritis in the Japanese population. Ann Rheum Dis 2020;79:1-9.

7 Catlin BW. Gardnerella vaginalis: characteristics, clinical considerations, and controversies. Clin Microbiol Rev 1992;5:213-37.

8 Rosca AS, Castro J, Sousa LGV, et al. Gardnerella and vaginal health: the truth is out there. FEMS Microbiol Rev 2019. doi:10.1093/femsre/fuz027. [Epub ahead of print: 07 Nov 2019].

9 Pybus V, Onderdonk AB. Evidence for a commensal, symbiotic relationship between Gardnerella vaginalis and Prevotella bivia involving ammonia: potential significance for bacterial vaginosis. J Infect Dis 1997;175:406-13.

10 Yurkovetskiy L, Burrows M, Khan AA, et al. Gender bias in autoimmunity is influenced by microbiota. Immunity 2013;39:400-12.

11 Markle JGM, Frank DN, Mortin-Toth S, et al. Sex differences in the gut microbiome drive hormone-dependent regulation of autoimmunity. Science 2013;339:1084-8. 